\title{
Actividad molusquicida de Laguncularia racemosa (L) C.F. Gaerth sobre Galba cubensis (Pfeiffer, 1839)
}

\section{Molluscicidal activity of Laguncularia racemosa (L) C.F. Gaerth on Galba cubensis (Pfeiffer, 1839)}

\author{
Luis José Rangel-Ruiz*, Luis Alfredo Cruz-Morales, Juan Armando Arévalo-de la Cruz, Jaquelina \\ Gamboa-Aguilar, Eduardo Moguel-Ordoñez, Coral Jazvel Pacheco-Figueroa, Luis Fernando Roa-de la Fuente \\ ${ }^{1}$ División Académica de Ciencias Biológicas. Universidad Juárez Autónoma de Tabasco. Carretera Villahermosa-Cárdenas Km. \\ 0.5 entronque con Saloya, CP. 94250, Villahermosa, Tabasco, México. \\ *Autor de correspondencia: ljrangel@msn.com
}

Artículo científico recibido: 01 de diciembre de 2015, aceptado: 30 de agosto de 2016

RESUMEN. Se evaluó la eficiencia molusquicida de Laguncularia racemosa sobre Galba cubensis utilizando dos extractos acuosos y aplicación directa de polvo (ADP), los extractos acuosos se elaboraron por el método de reposo en crudo (EMRC) y de infusión (EMI). Se utilizó un diseño al azar con tres tratamientos, un testigo y cinco repeticiones. En la determinación de la $\mathrm{DL}_{50}$ y $\mathrm{DL}_{90}$ se utilizó el programa Probit-Log. Se obtuvo la TL50 y TL90; las concentraciones del EMRC fueron de 500, 400, 333 y $289 \mathrm{~g} \mathrm{~L}^{-1}$; para el EMI de 50, 25, 12.5 y $6 \mathrm{~g} \mathrm{~L}^{-1}$; y para la ADP de $0.540,0.270$ y $0.135 \mathrm{~g}$. Se determinó la eficacia de los tratamientos y la residualidad del EMl a $50 \mathrm{~g} \mathrm{~L}^{-1}$. Para el EMRC las mortalidades acumuladas fueron de 100, 96, 90 y $66 \%$ con una $\mathrm{DL}_{50}$ de $267.56 \mathrm{~g} \mathrm{~L}^{-1}$ y $\mathrm{DL}_{90}$ de $392.68 \mathrm{~g} \mathrm{~L}^{-1}$. Para el EMI fue del $100,98,92$ y $20 \%$, con $\mathrm{DL}_{50}$ de $8.08 \mathrm{~g} \mathrm{~L}^{-1}$ y $\mathrm{DL}_{90}$ de $12.78 \mathrm{~g} \mathrm{~L}^{-1}$; para la ADP fue del 50, 48 y $48 \%$; solo la concentración de $0.540 \mathrm{~g}$ alcanzo el $50 \%$, la $\mathrm{DL}_{50}$ fue de $1.0 \mathrm{~g}$. Para el EMRC el TL90 se presentó a las 18,72 y $96 \mathrm{~h}$ para 500, 400, $333 \mathrm{~g} \mathrm{~L}^{-1}$; para el EMI a las $2 \mathrm{~h}$ para $50,25 \mathrm{~g} \mathrm{~L}^{-1}$ y $14 \mathrm{~h}$ en $12.5 \mathrm{~g}$ $\mathrm{L}^{-1}$. La residualidad mostro una baja persistencia de la toxicidad sobre el sustrato.

Palabras clave: Actividad Molusquicida, Laguncularia racemosa, Galba cubensis

ABSTRACT. The molluscicidal efficiency of Laguncularia racemosa on Galba cubensis was assessed using two aqueous extracts and direct application of powder (DAP). The aqueous extracts were prepared by the raw method extract (RME) and infusion method extract (IME) procedures. A randomized design with three treatments, one control and five replicates was used. For determining $L D_{50}$ and $L D_{90}$, the Probit-Log program was used. $L T_{50}$ and $L T_{90}$ were obtained; the RME concentrations were 500, 400, 333 and $289 \mathrm{~g} \mathrm{~L}^{-1}$; for IME, 50, 25, 12.5 and $6 \mathrm{~g} \mathrm{~L}^{-1}$; and for DAP, 0.540 , 0.270 and $0.135 \mathrm{~g}$. The efficacy of the treatments and the residuality of the IME at $50 \mathrm{~g} \mathrm{~L}^{-1}$ were determined. For RME, the cumulative mortalities were $100,96,90$ and $66 \%$ with a $\mathrm{LD}_{50}$ of $267.56 \mathrm{~g} \mathrm{~L}^{-1}$ and $\mathrm{LD}_{90}$ of $392.68 \mathrm{~g} \mathrm{~L}^{-1}$. For IME, it was 100, 98, 92 and $20 \%$, with $\mathrm{LD}_{50}$ of $8.08 \mathrm{~g} \mathrm{~L}^{-1}$ and $\mathrm{LD}_{90}$ of $12.78 \mathrm{~g} \mathrm{~L}^{-1}$; for DAP, it was 50,48 and $48 \%$; only the concentration of $0.540 \mathrm{~g}$ reached $50 \%$; the $\mathrm{LD}_{50}$ was $1.0 \mathrm{~g}$. For RME, $\mathrm{LT}_{90}$ occurred at 18,72 and $96 \mathrm{~h}$ for 500, 400, $333 \mathrm{~g} \mathrm{~L}^{-1}$; for IME, at $2 \mathrm{~h}$ for $50,25 \mathrm{~g} \mathrm{~L}^{-1}$ and $14 \mathrm{~h}$ for $12.5 \mathrm{~g} \mathrm{~L}^{-1}$. Residuality showed a low persistence of toxicity on the substrate.

Key words: Molluscicidal activity, Laguncularia racemosa, Galba cubensis.

\section{INTRODUCCIÓN}

La Fasciolosis, es una enfermedad helmíntica distribuida a nivel mundial que afecta al ganado bovino, ovino y caprino, así como al hombre y otros mamíferos (Olaechea 2004). Para completar su ciclo biológico Fasciola hepatica necesita dos hospedadores, uno intermediario, un caracol de la familia Lymnaeidae, y otro definitivo, que es un mamífero (Cruz 2011). La Fasciolosis es 
una de las enfermedades parasitarias más importantes, que provoca pérdidas económicas para los ganaderos, ocasionando baja producción y mala calidad de leche, reduce la tasa de crecimiento, ocasiona trastornos reproductivos, y la mortalidad. Los moluscos dulceacuícolas representan el eslabón más débil de la cadena de transmisión de muchos parásitos que requieren hospedadores intermediaros como la $F$. hepatica. Para el control de la parasitosis, lo recomendable es la disminución de las poblaciones de moluscos, lo que reduce la probabilidad de infección y transmisión al hospedero definitivo (Wong et al. 2010).

Para el control de los hospederos intermediarios los métodos más utilizados han sido los químicos inorgánicos y orgánicos (Becerra 2001). Debido a que los molusquicidas de origen sintéticos provocan la alteración de la estructura del medio ambiente, actuando como biocidas no específicos, con alto costo (Lannacone et al. 2008, Belete 2015, Njeh et al. 2016). En las tres últimas décadas se ha incrementado el interés en el campo de los productos naturales, lo que ha estimulado la búsqueda de nuevos compuestos bioactivos para aplicaciones farmacéuticas o agroquímicas, como insecticidas, acaricidas, fungicidas, herbicidas, molusquicidas, entre otros, como loa extractos de $L$. racemosa y R. mangle (Alemán et al. 2011, Regalado et al. 2016).

En el estado de Tabasco, las dos especies de mangle con mayor distribución y abundancia son $L$. racemosa y $R$. mangle (CONABIO 2009, CONABIO-CONANP 2009). Estas dos especies de mangle tienen alto contenido de taninos, que constituyen los componentes mayoritarios de $R$. mangle (80 \%), por lo que ha sido utilizado para la obtención de fitofármacos debido a sus propiedades medicinales que posee (Alemán et al. 2011, Regalado et al. 2016). Al respecto Rangel et al. (2016) probaron la eficiencia de extractos obtenidos por el método de reposo en crudo (EMRC), por infusión (EMI) y aplicación directa del polvo (ADP) de $R$. mangle sobre $G$. cubensis, obteniendo los mejores resultados para el EMI, con $\mathrm{DL}_{50}$ a $8.57 \mathrm{~g}$ $\mathrm{L}^{-1}$ y $\mathrm{DL}_{90}$ a $15.83 \mathrm{~g} \mathrm{~L}^{-1}$. El objetivo del presente trabajo es el evaluar la eficiencia molusquicida de $L$. racemosa sobre $G$. cubensis con dos extractos acuosos y una aplicación directa del polvo.

\section{MATERIALES Y MÉTODOS}

La colecta de $G$. cubensis se realizó de septiembre a noviembre de 2012, en el Rancho La Nueva Luz, en la ranchería Jahuacapa del municipio de Jalapa, Tabasco. Ubicado entre los $17^{\circ} 44^{\prime}$ $25.76^{\prime \prime}$ LN y los $92^{\circ} 50^{\prime} 25.25^{\prime \prime}$ LO. Los caracoles se recolectaron mediante colecta libre, el sustrato usado como sustrato en los terrarios fue sedimento y algas colectado del medio. Los terrarios para aclimatación de los caracoles fueron tinas de un $1 \mathrm{~m}^{3}$.

Se recolectaron $10 \mathrm{~kg}$ de hoja de $L$. racemosa en la localidad Arroyo Polo en el municipio de Centla, Tabasco, ubicado entre los $18^{\circ} 30^{\prime} 10.99^{\prime \prime}$ LN y los $92^{\circ} 39^{\prime} 3.64^{\prime \prime}$ LO. L. racemosa es un arbusto o árbol de hasta $20 \mathrm{~m}$ de alto, con $60 \mathrm{~cm}$ de diámetro de tallo a la altura del pecho. Su tronco es recto con ramas ascendentes, copa redonda y densa. Su distribución en México es de Tamaulipas a Yucatán por el Golfo de México, y de Baja California hasta Chiapas por el Océano Pacifico (CONABIOCONANP 2009).

Para la obtención del EMRC a concentración de $500 \mathrm{~g} \mathrm{~L}^{-1}$, se pesaron $5 \mathrm{~kg}$ de hoja y se licuaron en $10 \mathrm{~L}$ de agua, el extracto se dejó reposar por cinco días, trascurrido el tiempo se retiró el material vegetal por filtrado. Para obtener las concentraciones de 400,333 y $286 \mathrm{~g} \mathrm{~L}^{-1}$, se realizaron las diluciones correspondientes. Para el EMI se pesaron $1.5 \mathrm{Kg}$ de hojas, las cuales se deshidrataron a temperatura de $60 \pm 2{ }^{\circ} \mathrm{C}$ por $24 \mathrm{~h}$, para luego triturarlas en un molino casero, y mezclar $50 \mathrm{~g}$ del polvo en $1.0 \mathrm{~L}$ de agua, que se puso a hervir por 10 min, y dejó enfriar a temperatura ambiente. Cuando el extracto se enfrio, se filtró para obtener la concentración de $50 \mathrm{~g} \mathrm{~L}^{-1}$, de la misma manera se repitió el procedimiento para obtener las concentraciones de $25,12.5$ y $6 \mathrm{~g} \mathrm{~L}^{-1}$. Para la ADP se pesaron $0.135,0.270$ y $0.540 \mathrm{~g}$ de las hojas molidas para aplicarlas de forma directa.

Se determinó el $\mathrm{pH}$, temperatura y salinidad 


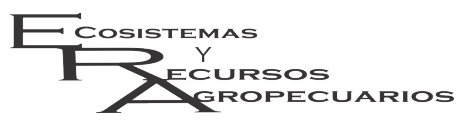

de los extractos y el polvo de $L$. racemosa. La toxicidad de los extractos EMRC y EMI se realizó bajo un diseño completamente al azar, con cuatro concentraciones y un testigo con cinco repeticiones. Para la ADP se probaron solo tres concentraciones. Para la evaluación del EMRC, EMI y ADP se elaboraron 75 terrarios en charolas de plástico de $15 \mathrm{~cm}$ de diámetro, a los cuales se les agregaron $200 \mathrm{~g}$ de sustrato esterilizado, y se saturo con agua declorinada con el fin de simular el hábitat donde se encuentran los caracoles. En cada terrario se colocaron 10 caracoles y se aplicaron $3 \mathrm{ml}$ de los extractos acuosos con un aspersor, y $0.135,0.270$ y $0.540 \mathrm{~g}$ para la aplicación directa del polvo. Después de $2 \mathrm{~h}$ post tratamiento y luego cada $2 \mathrm{~h}$ se registró el número de caracoles vivos y muertos. Como referencia de mortalidad se tomó el movimiento del cuerpo ante la luz y al tacto con una aguja de disección.

\section{Eficacia de los tratamientos}

La eficacia de cada tratamiento se calculó por la comparación del número de caracoles vivos y muertos, con el número de caracoles del grupo control. Para lo cual se realizaron evaluaciones cada $2 \mathrm{~h}$ hasta las $24 \mathrm{~h}$ y luego a las 48,72 y $96 \mathrm{~h}$, de acuerdo con Abbott (1987) y Otranto et al. (2005). Para lo cual se aplicó la siguiente formula: Porcentaje de eficacia $=$ (número promedio de caracoles vivos en el lote control - número promedio de caracoles vivos en los lotes tratados) / número promedio de caracoles vivos en el lote control) X 100 .

\section{Residualidad del extracto acuoso de EMI a 50 $\mathrm{g} \mathrm{L}^{-1}$ de $L$. racemosa en el sustrato}

Para determinar el tiempo en el que el extracto acuoso por infusión de $L$. racemosa obtenido por infusión a $50 \mathrm{~g} \mathrm{~L}^{-1}$, provoca una mortalidad superior al $90 \%$ de los caracoles, se evaluó su residualidad después de 2, 4, 6, 8 y 10 h de la aplicación y se comparó con el testigo de sustrato y agua destilada (Arcila et al. 2013). La mortalidad se determinó cada $2 \mathrm{~h}$ hasta las $10 \mathrm{~h}$.

\section{Análisis estadísticos}

Para establecer las diferencias entre las con- centraciones, se aplicó la prueba no paramétrica de Kruskal Wallis y la prueba de Rangos Múltiples con el programa estadístico de Statgraphics Centurión $X V$. Para establecer las diferencias de la efectividad de la residualidad del extracto en el sedimento, se aplicó un ANOVA Factorial $(\mathrm{p} \leq 0.05)$. Para determinar la $\mathrm{DL}_{50}$ y $\mathrm{DL}_{90}$ se utilizó regresión Probit-Log, con el programa SPSS Versión 20. El tiempo letal TL50 y TL90 para cada concentración se consideró, como el tiempo que tarda en morir el 50 y $90 \%$ de los caracoles, respectivamente.

\section{RESULTADOS}

Los extractos acuosos de L. racemosa y la aplicación en polvo tuvieron diversos grados de toxicidad para G. cubensis. Para el EMRC la mortalidad acumulada a las $96 \mathrm{~h}$ fue del 100, 96, 90 y 66 $\%$ para las concentraciones de 500, 400, 333 y 286 $\mathrm{g} \mathrm{L}^{-1}$, respectivamente. Presentando diferencias significativas solo la concentración de $286 \mathrm{~g} \mathrm{~L}^{-1}$ $(P=0.0017)$. De acuerdo con el análisis ProbitLog la $\mathrm{DL}_{50}$ se obtuvo a concentración de 267.559 $\mathrm{g} \mathrm{L}^{-1}$ y la $\mathrm{DL}_{90}$ a $392.683 \mathrm{~g} \mathrm{~L}^{-1}$ (Figura 1). El TL50 se presentó a las $10,14,16$ y $48 \mathrm{~h}$ para las concentraciones de 500, 400, 333 y $286 \mathrm{~g} \mathrm{~L}^{-1}$, respectivamente; y el TL90 a las 18,72 y 96 para las concentraciones de 500, 400, $333 \mathrm{~g} \mathrm{~L}^{-1}$ (Tabla 1).

El EMI presento una mortalidad acumulada a las $96 \mathrm{~h}$ de 100, 98, 92 y $20 \%$ para las concentraciones de $50,25,12.5$ y $6 \mathrm{~g} \mathrm{~L}^{-1}$, respectivamente; presentando diferencias significativas las concentraciones de 6 y $12.5 \mathrm{~g} \mathrm{~L}^{-1}(\mathrm{P}=0.0020)$ contra las de 50 y $25 \mathrm{~g} \mathrm{~L}^{-1}$. Para este extracto la $\mathrm{DL}_{50}$ fue de $8.076 \mathrm{~g} \mathrm{~L}^{-1}$ y la $\mathrm{DL}_{90}$ de $12.776 \mathrm{~g} \mathrm{~L}^{-1}$ (Figura 2). El TL90 se presentó a las $2 \mathrm{~h}$ para las concentraciones de 50 y $25 \mathrm{~g} \mathrm{~L}^{-1}$ y a las $14 \mathrm{~h}$ en la de 12.5 $\mathrm{g} \mathrm{L}^{-1}$. Hasta las $96 \mathrm{~h}$ la concentración de $6 \mathrm{~g} \mathrm{~L}^{-1}$ no alcanzo la TL50 (Tabla 1).

La aplicación directa en polvo (ADP), tuvo una mortalidad acumulada a las $96 \mathrm{~h}$ de 50,48 y 48 $\%$ para las concentraciones de $0.540,0.270$ y 0.135 $\mathrm{g}$, respectivamente; no presentándose diferencias 


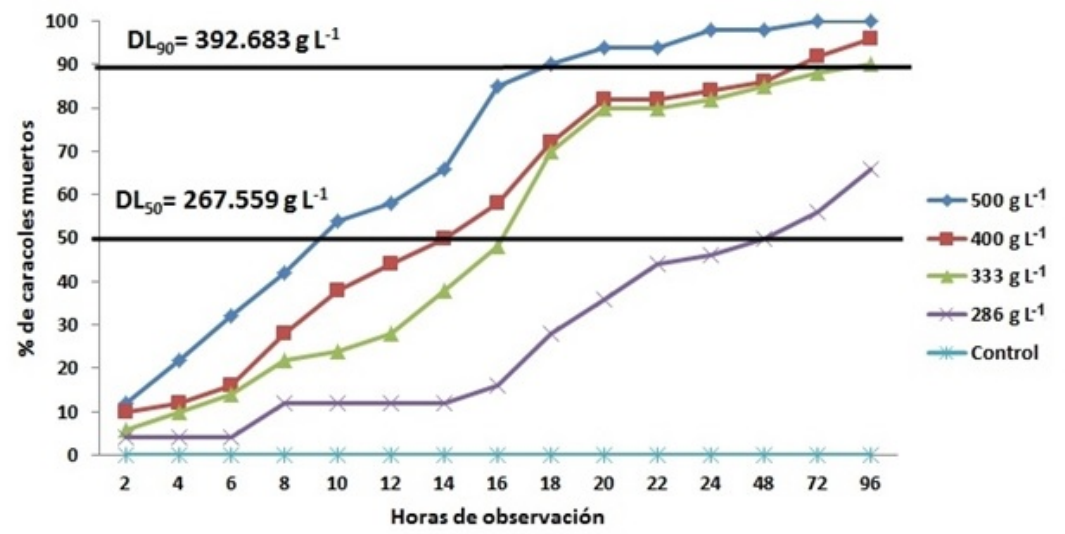

Figura 1. Mortalidad de G. cubensis por la aplicación del extracto acuoso de L. racemosa obtenido por el método de reposo en crudo.

Tabla 1. Tiempos letales de dos extractos acuosos y aplicación directa de polvo de $L$. racemosa sobre G. cubensis.

\begin{tabular}{|c|c|c|c|c|}
\hline Método & Concentración & TL50 (h) & TL90 (h) & TL100 (h) \\
\hline Método de extracción por infusión & $50.0 \mathrm{~g} \mathrm{~L}^{-1}$ & 2 & 2 & 2 \\
\hline \multirow[t]{3}{*}{$(\mathrm{MEI})$} & $25.0 \mathrm{~g} \mathrm{~L}^{-1}$ & 2 & 2 & - \\
\hline & $12.5 \mathrm{~g} \mathrm{~L}^{-1}$ & 2 & 14 & - - \\
\hline & $6.0 \mathrm{~g} \mathrm{~L}^{-1}$ & - & - - & - - \\
\hline \multirow{4}{*}{$\begin{array}{l}\text { Método de extracción por reposo en } \\
\text { crudo (MERC) }\end{array}$} & $500 \mathrm{~g} \mathrm{~L}^{-1}$ & 10 & 18 & 72 \\
\hline & $400 \mathrm{~g} \mathrm{~L}^{-1}$ & 14 & 62 & - - \\
\hline & $333 \mathrm{~g} \mathrm{~L}^{-1}$ & 16 & 90 & - - \\
\hline & $286 \mathrm{~g} \mathrm{~L}^{-1}$ & 48 & - - & - - \\
\hline \multirow[t]{3}{*}{ Aplicación en polvo (AP) } & $0.540 \mathrm{~g}$ & 96 & - & - \\
\hline & $0.270 \mathrm{~g}$ & - - & - - & - - \\
\hline & $0.135 \mathrm{~g}$ & - - & -- & - - \\
\hline
\end{tabular}

significativas entre ellas $(P=0.0682)$. La $D_{50}$ se tuvo a una concentración de $1.0 \mathrm{~g}$ y nunca se alcanzó la $\mathrm{DL}_{90}$, la cual de acuerdo al análisis Probit-Log se debe presentar en una concentración de $648.941 \mathrm{~g}$ (Figura 3). Mientras que la TL50 se tuvo a las $72 \mathrm{~h}$ (Tabla 1 ).

\section{Eficacia de los tratamientos}

La mayor eficacia para los tratamientos del EMRC la tuvo la concentración de $500 \mathrm{~g} \mathrm{~L}^{-1}$ con el $100 \%$ a las $72 \mathrm{~h}$ de la aplicación; siguida de la concentración de $400 \mathrm{~g} \mathrm{~L}^{-1}$ con $96 \%$ de eficacia a las 96 h; mientras que la concentración de $333 \mathrm{~g}$ $\mathrm{L}^{-1}$ tuvo una eficacia del $90 \%$ a las $96 \mathrm{~h}$. Para los tratamientos del EMI la concentración de $50 \mathrm{~g} \mathrm{~L}^{-1}$ tuvo el $100 \%$ de eficacia a las $2 \mathrm{~h}$; siguido de la concentración de $25 \mathrm{~g} \mathrm{~L}^{-1}$ con el $98 \%$ de eficacia a las $2 \mathrm{~h}$; mientras que las concentraciones de 12.5 y $6 \mathrm{~g} \mathrm{~L}^{-1}$ tivieron una eficacia del $92 \%$ a las $14 \mathrm{~h}$ y del $20 \%$ a las $24 \mathrm{~h}$, respectivamente. Para la AP, ninguna concentración supero el $90 \%$ (Tabla 2). Debido a que en los bioensayos no se encontraron mortalidades diferentes a cero en ninguno de los lotes testigo, los resultados de eficacia corresponden a mortalidad promedio a las $96 \mathrm{~h}$ de exposición.

\section{Evaluación de la residualidad del EMI a $50 \mathrm{~g}$ $\mathbf{L}^{-1}$}

La residualidad del EMI a $50 \mathrm{~g} \mathrm{~L}^{-1}$ tuvo baja persistencia de toxicidad sobre el sustrato. La mortalidad de G. cubensis fue del $100 \%$ a las $2 \mathrm{~h}$ y del $58 \%$ a las 4 h (Tabla 3); mientras que el testigo no 
Tabla 2. Eficacia de los extractos EMRC, EMI y AP de L. racemosa sobre G. cubensis.

\begin{tabular}{rrrrrrrrrrrr}
\hline \multirow{2}{*}{ Horas } & \multicolumn{1}{c}{ EMRC $\left(\mathrm{g} \mathrm{L}^{-1}\right)$} & \multicolumn{1}{c}{ Porcentaje de eficacia } \\
\cline { 2 - 13 } & 500 & 400 & 333 & 286 & 50 & 25 & 12.5 & 6 & 0.540 & 0.270 & 0.135 \\
\hline 2 & 12 & 10 & 6 & 4 & 100 & 98 & 88 & 0 & 0 & 0 & 0 \\
4 & 22 & 12 & 10 & 4 & 100 & 98 & 88 & 0 & 0 & 0 & 0 \\
6 & 32 & 16 & 14 & 4 & 100 & 98 & 88 & 0 & 10 & 0 & 0 \\
8 & 42 & 28 & 22 & 12 & 100 & 98 & 88 & 0 & 10 & 4 & 0 \\
10 & 54 & 38 & 24 & 12 & 100 & 98 & 88 & 0 & 20 & 4 & 2 \\
12 & 58 & 44 & 28 & 12 & 100 & 98 & 88 & 0 & 28 & 4 & 2 \\
14 & 66 & 50 & 38 & 12 & 100 & 98 & 92 & 0 & 28 & 8 & 2 \\
16 & 85 & 58 & 48 & 16 & 100 & 98 & 92 & 0 & 32 & 8 & 6 \\
18 & 90 & 72 & 70 & 28 & 100 & 98 & 92 & 0 & 32 & 8 & 6 \\
20 & 92 & 82 & 80 & 44 & 100 & 98 & 92 & 0 & 32 & 20 & 10 \\
22 & 94 & 82 & 80 & 44 & 100 & 98 & 92 & 10 & 32 & 20 & 10 \\
24 & 98 & 84 & 82 & 46 & 100 & 98 & 92 & 20 & 34 & 42 & 14 \\
48 & 98 & 86 & 85 & 50 & 100 & 98 & 92 & 20 & 48 & 48 & 38 \\
72 & 100 & 92 & 88 & 56 & 100 & 98 & 92 & 20 & 50 & 48 & 48 \\
96 & 100 & 96 & 90 & 66 & 100 & 98 & 92 & 20 & 50 & 48 & 48 \\
\hline
\end{tabular}

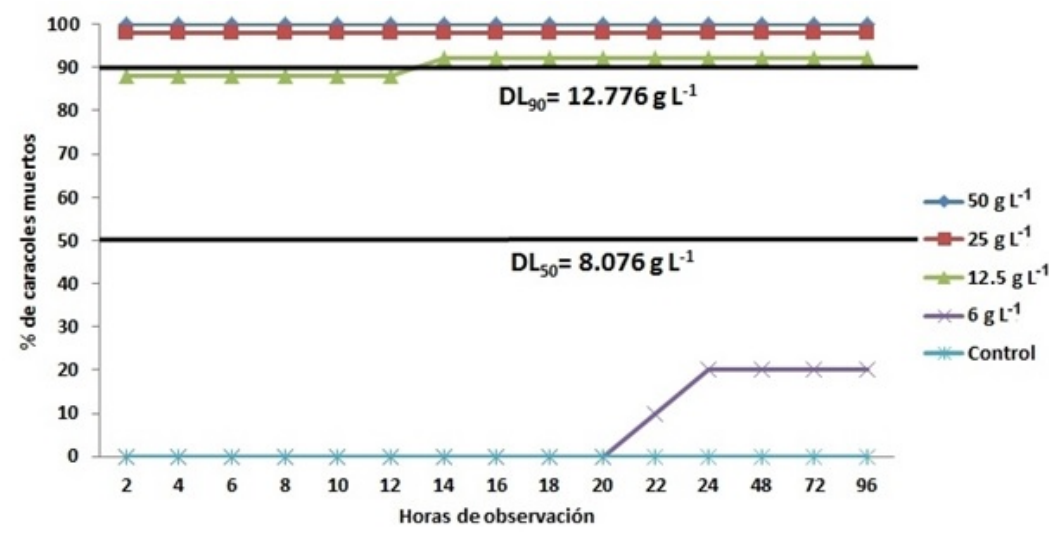

Figura 2. Mortalidad de G. cubensis por la aplicación del extracto acuoso de L. racemosa obtenido por el método de infusión.

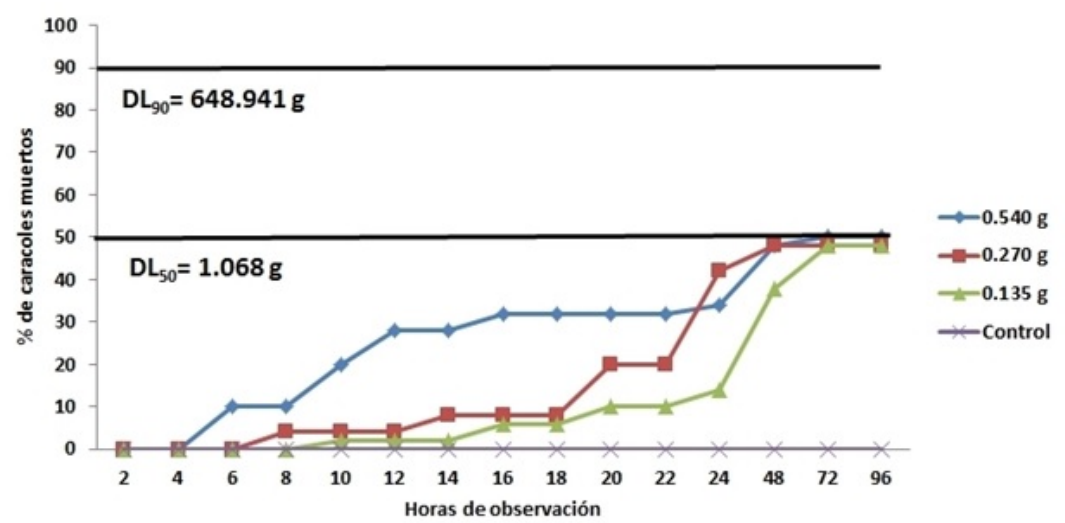

Figura 3. Mortalidad de G. cubensis por la aplicación directa de polvo de $L$ racemosa. 
Tabla 3. Residualidad del extracto acuoso de $L$. racemosa por el EMI a $50 \mathrm{~g} \mathrm{~L}^{-1}$.

\begin{tabular}{|c|c|c|c|c|}
\hline No & Descripción & Concentración & Hora de introducción & Promedio de mortalidad \\
\hline 1 & L. racemosa & $50 \mathrm{~g} \mathrm{~L}^{-1}$ & 2 & $10.0 \mathrm{a}$ \\
\hline 2 & L. racemosa & $50 \mathrm{~g} \mathrm{~L}^{-1}$ & 4 & $5.8 \mathrm{~b}$ \\
\hline 3 & L. racemosa & $50 \mathrm{~g} \mathrm{~L}^{-1}$ & 6 & $1.8 \mathrm{c}$ \\
\hline 4 & L. racemosa & $50 \mathrm{~g} \mathrm{~L}^{-1}$ & 8 & $1.2 \mathrm{c}$ \\
\hline 5 & L. racemosa & $50 \mathrm{~g} \mathrm{~L}^{-1}$ & 10 & $0.0 \mathrm{~d}$ \\
\hline 6 & Control & $0 \mathrm{~g} \mathrm{~L}^{-1}$ & 0 & $0.0 \mathrm{~d}$ \\
\hline
\end{tabular}

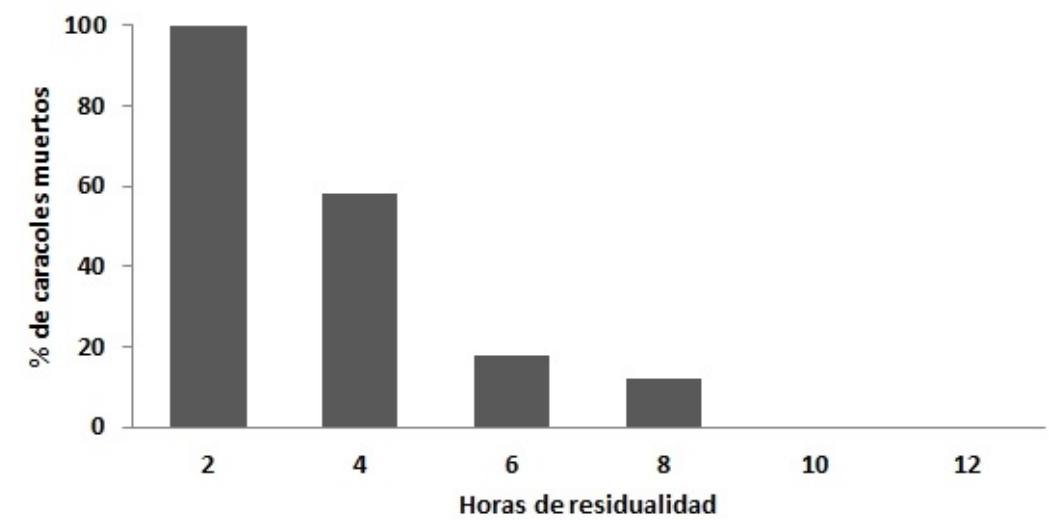

Figura 4. Residualidad del extracto por el método de infusión a $50 \mathrm{~g} \mathrm{~L}^{-1}$ de $L$. racemosa sobre G. cubensis.

tuvo mortalidad. La mortalidad promedio entre los lotes a las 2 y $4 \mathrm{~h}$ fueron diferentes, en los tiempos de 6,8 y $10 \mathrm{~h}(\mathrm{P}=0.0000)$, mientras que los lotes de los tiempos 5,6 y $8 \mathrm{~h}$ y el testigo no fueron diferentes $(p \geq 0.05)$ (Figura 4).

\section{DISCUSIÓN}

De los dos extractos acuosos, el EMI tuvo el mayor porcentaje de mortalidad, el cual fue superior al $90 \%$ en la concentración de $12.5 \mathrm{~g} \mathrm{~L}^{-1}$ a las 14 $\mathrm{h}$, seguida de la concentración de 25 y $50 \mathrm{~g} \mathrm{~L}^{-1}$ a las $2 \mathrm{~h}$. El EMRC tuvo tres concentraciones que superaron el $90 \%$ de mortalidad, con concentraciones que son superiores a las del EMI. Los resultados obtenidos por el método de eficacia son similares a los resultados obtenidos del análisis Probit-Log, que indican que se obtuvo una $\mathrm{DL}_{90}$ de $12.776 \mathrm{~g} \mathrm{~L}^{-1}$ a las $14 \mathrm{~h}$. Este mismo comportamiento se observó con el EMI del mangle rojo (Rhizophora mangle) en concentración $15.83 \mathrm{~g} \mathrm{~L}^{-1}$ en la $\mathrm{DL}_{90}$ (Rangel et al. 2016). La disminución de la residualidad del extracto a $50 \mathrm{~g} \mathrm{~L}^{-1}$ del 100 al $58 \%$ a las $4 \mathrm{~h}$, muestra una alta efectividad y baja persistencia. Por lo que la mejor opción para la aplicación en campo es la concentración de $50 \mathrm{~g} \mathrm{~L}^{-1}$ para tener el 100 $\%$ de mortalidad a las $2 \mathrm{~h}$ de exposición, por su mayor eficacia en el menor tiempo y baja residualidad. La eficiencia en los extractos de $L$. racemosa, probablemente se debe a su composición química de sus compuestos activos, como esteroides, taninos, antocianinas y saponinas (Bandaranayake 2002). Los taninos tienen interés especial como fitofármacos, debido a sus propiedades medicinales, también se aplican contra diferentes especies de helmintos como $F$. hepatica. Los cuales se encuentran en gran concentración en $L$. racemosa (Rangel et al. 2016), destacando la presencia de epicatequinas, catequinas, ácido clorogénico, ácido gálico, ácido egálico, galotaninos, elagitaninos y taninos condensados con actividad antiséptica, antibacteriana, antifúngica, entre otras (Alemán 2011). 
El tiempo de permanencia del extracto en condiciones de campo depende de las características del suelo en que se aplique, entre ellas el relieve, composición física y química, vegetación, permeabilidad, contenido de minerales, materia orgánica, proporción de arcilla, arena, grava y otros factores como el clima. El suelo en donde se colectaron los caracoles y de donde se tomó el sedimento para los cultivos y terrarios de prueba de este trabajo es de tipo fluvisol formado por sedimentos aluviales obscuros, de textura media a media-gruesa (franco arenoso a arcillosa) bien estructurada, con contenido medio de materia orgánica, con pendiente menor a $2 \%$ y sujeto a inundación por algunos días (Palma-López et al. 2007, Zavala Cruz et al. 2011). Es necesario tomar en cuenta que $L$. racemosa se encuentra sujeta a protección por la Norma Oficial Mexicana NOM-059-2010-SEMARNAT (SEMARNAT 2010) que la coloca en la categoría de amenazada, además de su protección por la NOM022-SEMARNAT-2003 (SEMARNAT 2003) por el peligro de desaparecer a corto o mediano plazo. Por lo anterior para el uso extensivo de las hojas de mangle blanco, estas se tienen que obtener de plantaciones especiales como Unidades de Manejo Forestal (CONAFOR 2013, Rangel et al. 2016).

\section{CONCLUSIONES}

El EMI a $50 \mathrm{~g} \mathrm{~L}^{-1}$ de $L$. racemosa es un buen molusquicida, eficaz en bajas concentraciones con rápida acción y baja persistencia, lo que evita efectos colaterales sobre la diversidad de organismos que habitan junto a $G$. cubensis. Crece en zonas costeras, tiene alta capacidad de regeneración de las partes utilizadas para la elaboración del extracto, por lo que no se daña la planta y los procedimientos de extracción son fáciles y económicos.

\section{AGRADECIMIENTOS}

A la Universidad Juárez Autónoma de Tabasco, al Consejo Nacional de Ciencia y Tecnología (CONACYT) y al Gobierno del estado de Tabasco por financiar el proyecto "Programa de Control para la Fasciolosis en el Estado de Tabasco" Clave de Registro TAB-2010-C23-149590.

\section{LITERATURA CITADA}

Abbott WS (1987) A method of computing the effectiveness of an insecticide. Journal American Mosquito Control Association 3: 302-303.

Alemán GY, Sánchez PLM, Rodríguez Y, Olivares JL, Rodríguez JG (2011) Actividad larvicida de extractos de Rhizophora mangle L. contra estrongílidos gastrointestinales de ovinos. Revista de Salud Animal 33: 111-115.

Arcila MA, Duarte CAF, Villalba GDA, Benavides MP (2013) Nuevo producto en el manejo integrado de la broca del café en Colombia. Avances Técnicos Cenicafe 437: 1-8.

Bandaranayake WM (2002) Bioactivities, bioactive compounds and chemical constituents of mangrove plants. Wetlands Ecology and Management 10: 421-452.

Becerra RWM (2001) Consideraciones sobre estrategias sostenibles para el control de Fasciola hepatica en Latinoamerica. Revista Colombiana de Ciencias Pecuarias 14: 28-35.

Belete EM (2015) Schistosomiasis control strategies, with emphasis on snail control using molluscicides. International Journal of Health Sciences and Research 5: 572-584

CONABIO (2009) Manglares de México: Extensión y distribución. 2a ed. Comisión Nacional para el Conocimiento y Uso de la Biodiversidad. México. 99p. 
CONABIO-CONANP (2009) Mangle blanco (Laguncularia racemosa). http://www.biodiversidad.gob.mx/v ingles/species/especies_priori/fichas/pdf/Mangleblanco02jul09.pdf. Fecha de consulta 22 de julio de 2016.

CONAFOR (2013) Ley General de Desarrollo Forestal Sustentable. Documento disponible en http://www.cona for.gob.mx/portal/docs/subsecciones/normateca/LGDFS.pdf. Fecha de consulta 22 de julio de 2016.

Cruz MI (2011) Epidemiología y control de los huéspedes intermediarios de Fasciola hepatica. En: Quiroz RH, Figueroa CJA, Ibarra VF, López AME (ed.). Epidemiologia de enfermedades parasitarias en animales domésticos. UNAM. México, pp: 173-207

Lannacone J, Alvariño L, Pérez D (2008) Efecto de Paullinia clavigera "Sacha yoco" (Sapindaceae) sobre la eclosión de huevos de Fasciola hepatica. Neotropical Helminthology 2: 54-60.

Njeh F, Feki H, Koubaa I, Hamed N, Damak M, Ayadi A, et al. (2016) Molluscicidal activity of Solanum elaeagnifolium sedes against Galba truncatula intermediate host of Fasciola hepatica: Identification of $\beta$-solamarine. Pharmaceutical Biology 54: 726-731.

Olaechea V (2004) Epidemiología y control de Fasciola hepatica en Argentina. En: Nari A, Fiel C (ed.). Enfermedades parasitarias de importancia económica en bovinos. Hemisferio Sur. Uruguay. pp: 213233.

Otranto D, Lia RP, Cantacessi C, Galli G, Paradies P, Mallia E, et al. (2005) Efficacy of a combination of imidacloprid $10 \%$ /permethrin $50 \%$ versus fipronil $10 \% /(\mathrm{S})$-methoprene $12 \%$, against ticks in naturally infected dogs. Veterinary Parasitology 130: 293-304

Palma-López DJ, Cisneros DJ, Moreno-Cáliz E, Rincón-Ramírez JA (2007) Suelos de Tabasco: Su uso y manejo sustentable. Colegio de Postgraduados-ISPROTAB-FUPROTAB. Villahermosa, Tabasco, México. 195p.

Rangel RLJ, Arévalo CJA, González GG, Hernández AOJ, Gamboa AJ, Montiel JM, et al. (2016) Toxicological evaluation of three methods of applying Rhizophora mangle on Galba cubensis (Mollusca: Gastropoda: Lymnaeidae). Ecosistemas y Recursos Agropecuarios 3: 327-334.

Regalado AI, Sánchez LM, Mancebo B (2016) Rhizophora mangle L. (mangle rojo): Una especie con potencialidades de uso terapéutico. Journal of Pharmacy \& Pharmacognosy Research 4: 1-17.

SEMARNAT (2003) Norma Oficial Mexicana NOM-022-SEMARNAT-2003, que establece las especificaciones para la preservación, conservación, aprovechamiento sustentable y restauración de los humedales costeros en zonas de manglar. Diario Oficial de la Federación (DOF), jueves 10 de abril de 2003.

SEMARNAT (2010) Norma Oficial Mexicana NOM-059-SEMARNAT-2010. Protección ambiental, especies nativas de México de flora y fauna silvestres, categorías de riesgo y especificaciones para su inclusión, exclusión o cambio, Lista de especies en riesgo. Diario Oficial de la Federación (DOF), jueves 30 de diciembre de 2010.

Wong L, Vázquez A, Quesada M, Sánchez J, Hevia Y, Fuentes J, et al. (2010) Estudios ecológicos en moluscos de importancia médico-veterinaria en la granja de desarrollo La Coca. Revista Cubana de Medicina Tropical 62: 18-23.

Zavala-Cruz J, Palma-López DJ, Fernández-Cabrera CR, López Castañeda A, Torres SED (2011) Degradación y conservación de suelos en la cuenca del río Grijalva, Tabasco. Colegio de Postgraduados - Secretaría de Recursos Naturales y Protección Ambiental - PEMEX. Villahermosa, Tabasco, México. 90p. 